\title{
Short Circuit Fault Detection against High Thermal Background Using a Two-Level Scheme Based on DoG Filter
}

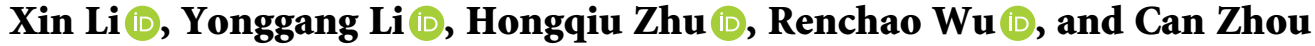 \\ School of Automation, Central South University, Changsha 410083, China \\ Correspondence should be addressed to Can Zhou; zhoucan@csu.edu.cn
}

Received 19 August 2020; Revised 24 December 2020; Accepted 18 January 2021; Published 29 January 2021

Academic Editor: Zhile Yang

Copyright (c) 2021 Xin Li et al. This is an open access article distributed under the Creative Commons Attribution License, which permits unrestricted use, distribution, and reproduction in any medium, provided the original work is properly cited.

\begin{abstract}
Short circuit is a key factor which drastically affects the efficiency of metal electrorefining. Infrared image of the intercell busbar region is used to perform short circuit detection. To cope with the high thermal background, a two-level short circuit detection method is designed. Firstly, with background subtraction, high intensity short circuit electrodes, as well as the background, are removed, and normal working electrodes are preserved. In the second stage, suspicious short circuit areas are sifted out by normal electrode detecting and texture period estimation. Gaussian difference filter (DoG) which is based on the human visual system is improved to match the target gray distribution. A comparative experiment indicates that the proposed orthogonal DoG outperforms the original DoG and top-hat in the accuracy of normal electrode detection. The two-level detection method in this paper is applied in a copper electrolysis plant and exhibits superiority in locating short circuits and avoiding miss detection.
\end{abstract}

\section{Introduction}

Electrorefining of copper, lead, and nickel is an important electrochemical process involving a mass of densely distributed electrodes as shown in Figure 1. Plate electrodes are inserted into the cell full of hot electrolyte, and the electrode conducting rods hang at the intercell busbar. Short circuits between electrodes can drastically decrease the current efficiency, the electrolyte stability, and the copper quality [1]. Executing large-scale manual inspection is time-consuming, and the electrolysis environment is harmful to human health. Therefore, online detection and fault diagnosis of the electrolytic cells are essential.

The infrared (IR) image method [2-4] has received more and more attention due to the capacity of large area monitoring. An IR image taken at the top of copper electrolysis cells in our work is shown in Figure 2. The image contains three electrolytic cells. Each cell is mainly composed of canvas area and intercell busbar area. Canvas region is often used as a region of interest (RoI) for fault detection $[3,4]$, as short circuit raises the temperature of electrode conducting rod that is under the canvas. However, missed detection often occurs, due to some random factors such as water accumulation on the canvas and the noncontact between the canvas and the electrode. The loss of production caused by missed detection is more serious than that caused by error detection. To avoid missed detection, the intercell busbar area is proposed as the RoI in this paper based on the temperature characteristics of the area.

However, the high thermal background and the compact electrode distribution pose challenges for high temperature short circuit target detection. The contrast between the background and the target is smoothed, making it difficult to distinguish the target directly [5]. Compact electrode distribution arises new challenges for the identification and location of short circuit electrodes.

Note that normal working suspenders on the busbar keep better contrast with the background. Combining this characteristic with the regular texture of the busbar area, we design a two-level detection scheme (Figure 3). The objective is to identify short circuit area by detecting the recognizable normal working suspenders. In the first stage, background subtraction is implemented with a median filter [6]. The aim is to remove the background as well as short circuit suspenders and preserve normal working suspenders. The 


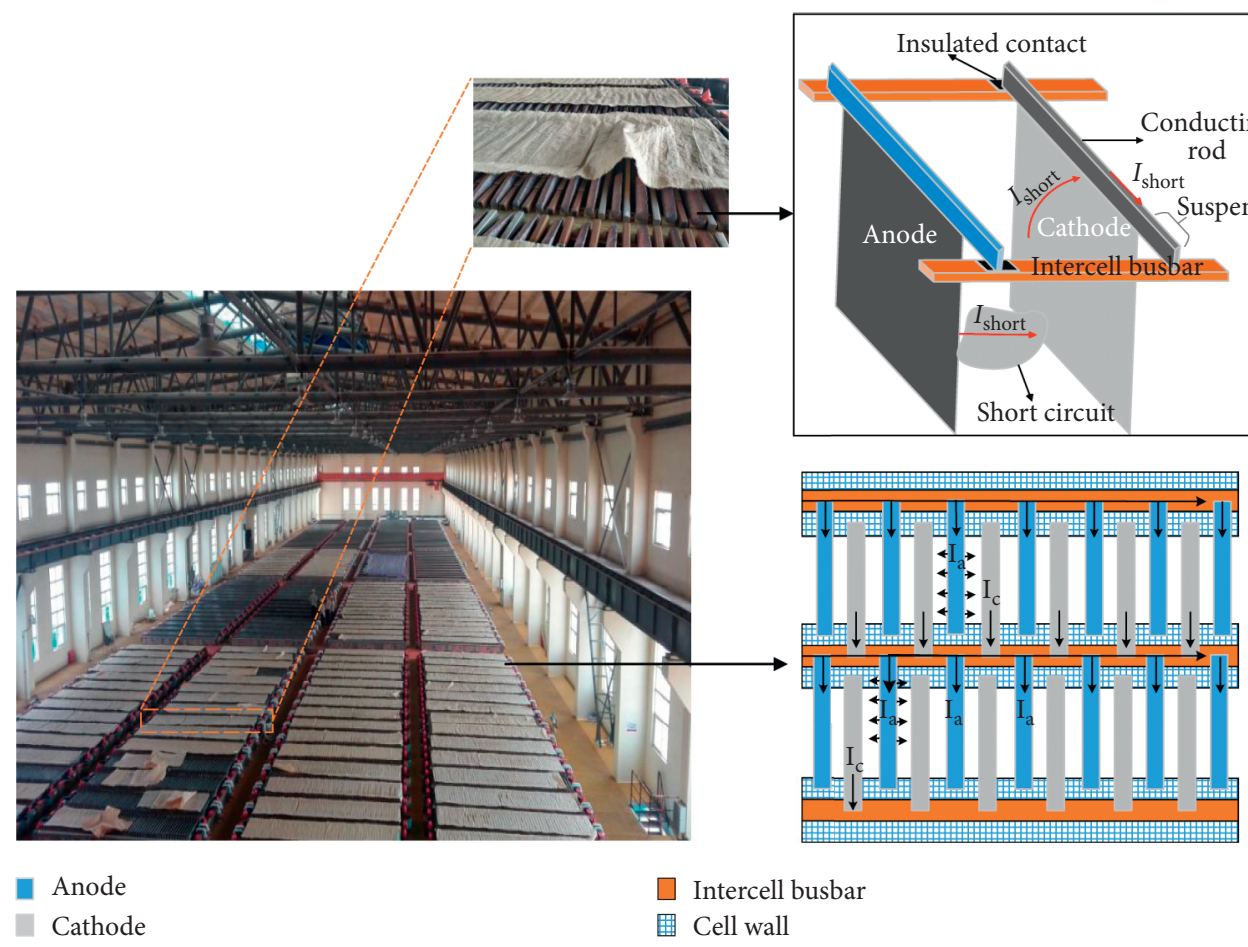

FIGURE 1: Overhead view of electrolytic cells and electric model superimposed over a physical diagram of the electrolytic refining process.

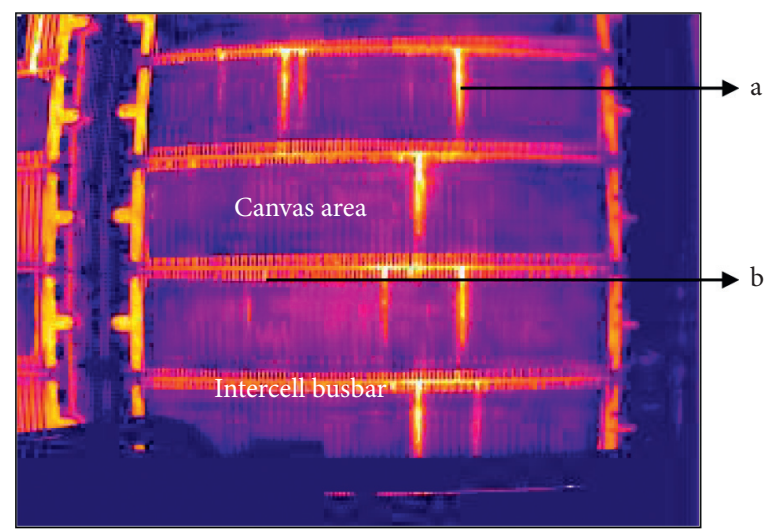

FIGURE 2: IR image of electrolytic cells taken at the top of cells surface: $\mathrm{a}$ is a common short circuit and $\mathrm{b}$ is a short circuit that has not caused the canvas temperature change.

realization of the idea benefits from that the temperature of short circuit suspenders is close to the background so that the gray intensity of the two are approximate. Although the background subtraction highlights these preserved normal working suspenders, these small and dim targets are still hidden in a heavy clutter due to the complex grayscale distribution of the intercell busbar area, which bring obstacles for detection. This is referred to as a small target detection problem [7-9]. In the second stage, we detect normal suspenders with an improved DoG filter which is based on the visual attention model. Incorporate the texture period of the busbar area into the short circuit diagnosis. If a distance between two located normal suspenders is larger than the texture period, the opening between two normal suspenders is diagnosed as a suspicious short circuit area.
This paper is organized as follows: Section 2 describes the thermal distribution of the intercell busbar and discusses the detection difficulties. In Section 3, details of the proposed two-level detection scheme and the improved DoG filter are presented. In Sections 4 and 5, experimental results and concluding remarks are reported.

\section{Related Work}

2.1. Thermal Distribution Characteristics of Intercell Busbar and Problem Description. IR image of the electrolytic cell is the result of resistance heat and heat conduction. As shown in Figure 1, the middle busbar receives parallel currents from cathode $\left(I_{a}\right)$ of a cell, rebalances the total current, and splits it to each anode branch $\left(I_{a}\right)$ of the next cell $[10,11]$. When 


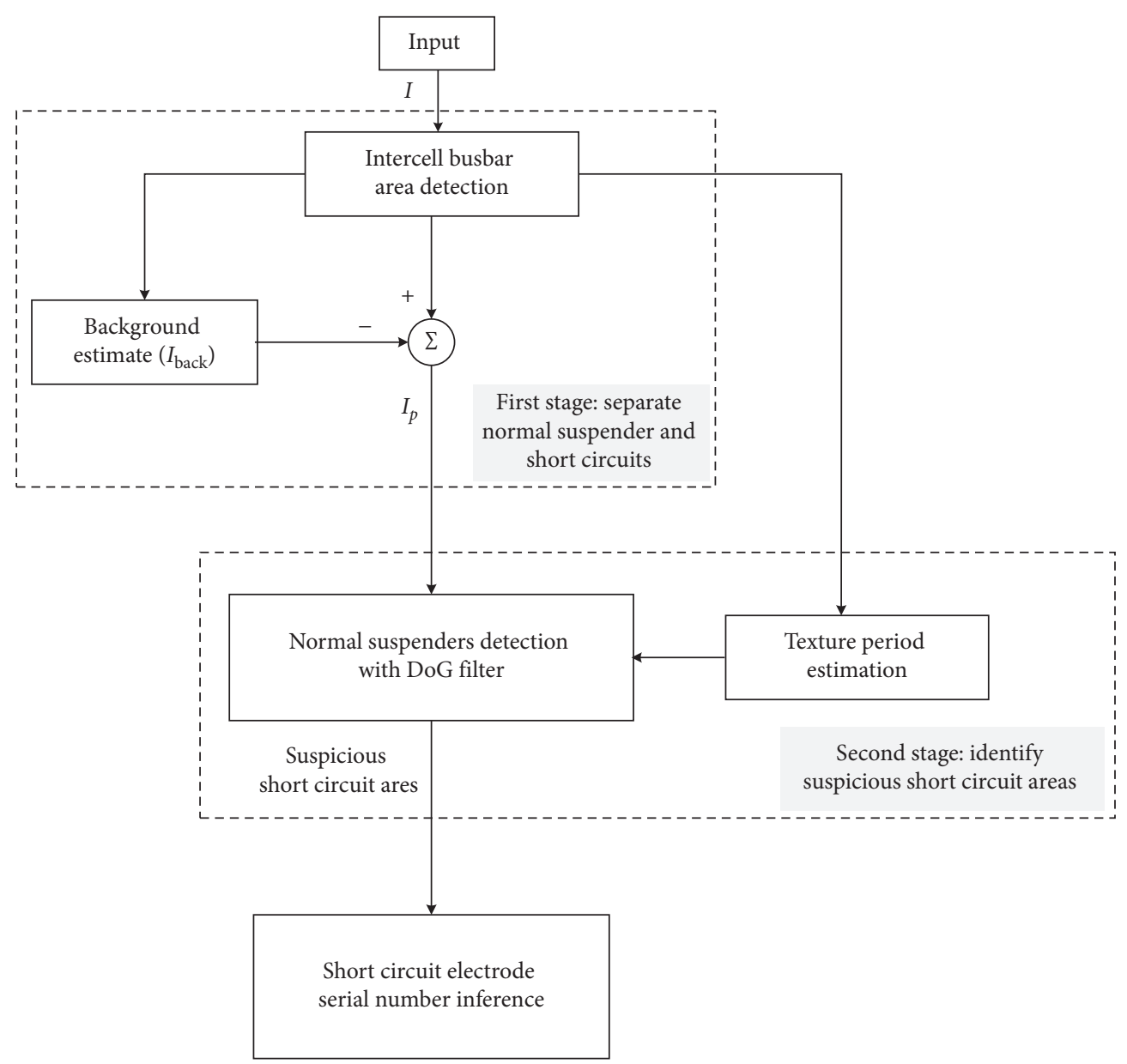

FIgURE 3: Two-level short circuit detection scheme flowchart.

working normally, the temperature of the busbar is higher than that of conducting rods [12]. Therefore, in the image, all electrode suspenders are against a background with high grayscale intensity.

After short circuiting, a large current flows through the suspender into the busbar. Compared with other parts of the rod, the connected suspenders temperature rises firstly $[13,14]$. Along with the time, the intensity of the fault suspenders gradually approaches that of the background and eventually exceeds it. Thus, the gray difference between the suspender and the background is weakened, and the suspender is blended into the background (Figure 4). Unlike short circuited suspenders, normal working suspenders keep better contrast with the background. Furthermore, all suspenders are evenly arranged on the busbar, like a row of fence. Among normal working suspenders, short circuited ones appeared as an opening of the fence.

Expect that the current on each electrode branch is equal. However, due to the complex electrochemical reaction, the currents of each electrode are various. The current will find its path of least resistance on the busbar. It causes nonuniform temperature distribution on the busbar, which further results in heavy background clutter on the image. Against the heavy clutter background, there are suspenders occupying a few pixels, which bring difficulties for detection.
2.2. Small Target Detection Based on the Human Visual System. Robust detection of small targets in a complex background is difficult due to the low signal-clutter ration (SCR) [15, 16]. Background suppression [17, 18] and highlight target $[19,20]$ are two mainstreams to solve the problem. These studies reveal that the background is spatially correlated, while the target and the background are spatially discontinuous. To synchronously suppress background clutters and enhance targets, an idea based on local difference is proposed [21]. It utilized a variable-size window to search the target. It is effective in the case of sparse distribution of targets, but not suitable when small targets are densely distributed. If the distance between two adjacent targets is less than the window size, the gray value of the adjacent target will reduce the local gray difference and affects the detection result. The human visual system [22] is also a local-based mechanism that utilizes visual attention to extract key information. The intensity distribution of a small target can refer to a Gaussian-like spot, with this assumption difference of Gaussian filter (DoG) proposed to accurately locate targets [23]. Through gray distribution pattern matching, the DOG filter can simultaneously suppress background and enhance targets. For the target with various scales, locations can be determined by searching the maximum response point in a continuous scale space [24]. The 


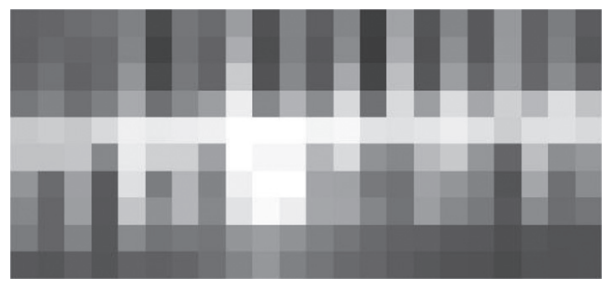

(a)

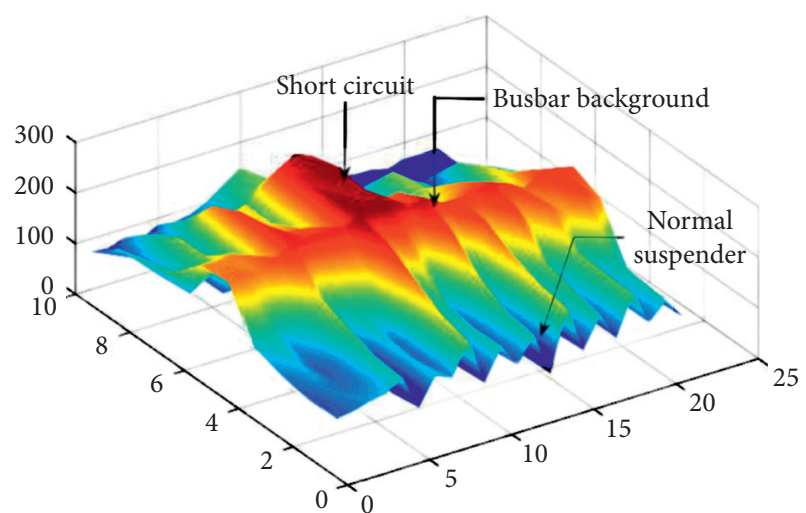

(b)

Figure 4: (a) Image patch of intercell busbar area. (b) 3D view of the image patch.

result is not affected by targets distribution. But suspender in our problem has a unique gray distribution pattern. To match suspenders better and improve the accuracy, we improve the DoG filters parameters. Based on the separability of the Gaussian convolution kernel, we fix the scale parameter of the row convolution kernel and only change the scale parameter of the vertical convolution kernel. Results show that the improved orthogonal DoG filter can effectively increase the detection rate of normal suspenders and further ensure the accuracy of short circuit diagnosis.

\section{Two-Level Short Circuit Detection Scheme}

3.1. First Stage: Background Removal. Suspenders are located near a horizontal line between two heterogeneous backgrounds, the canvas region and the busbar region, as shown in Figure 5. The heterogeneous background will increase the false alarm rate $[6,21]$. To preserve normal electrodes only and reduce the influence of the heterogeneous background, we adopt a one-dimensional median filter to model the background. Then, the background is subtracted from the original image. The one-dimensional median filter is transversal (equation (1)); in our work, we set $n=1$. The background removal process is shown in equation (2). As in our problem the normal suspenders gray value is lower than that of the background, to display it better the result is reversed:

$$
I_{\text {back }}=\operatorname{median}\{I(x-n, y), \ldots, I(x, y), \ldots, I(x+n, y)\},
$$

$$
I_{p}=-1 *\left(I-I_{\text {back }}\right)
$$

The estimated background of the intercell busbar and the result of background removal are shown in Figure 5. After background removal, all normal working suspenders are retained and enhanced. These normal suspenders are distributed regularly. At the original short circuit position, an opening is formed (Figure 5(c)). Next, we will detect these retained normal suspenders and identify the opening.

\subsection{Second Stage: Suspicious Short Circuit Area Identification}

3.2.1. Small Target Detection Using DoG Filter. The intensity distribution model of a small target is as the following equation, where $I_{\max }$ is the peak intensity of the target. $\left(x_{c}, y_{c}\right)$ denotes the central position of the target. $\sigma$ is the standard deviation and it determines the target size:

$T\left(x, y \mid x_{c} t, n y_{c} q, h \sigma\right)=I_{\max } \exp \left\{-\frac{\left(x-x_{c}\right)^{2}+\left(y-y_{c}\right)^{2}}{2 \sigma^{2}}\right\}$.

Laplacian of Gaussian (LoG) filter (equation (4)) [25] is the second derivative of Gaussian function (equation (5) and has a strong filtering response to the spot-like target. The LoG filter was introduced to enhance the gray difference between the small target and background [26]. Figure 6 [23] shows the distribution pattern of a small target and an LoG filter. The LoG operator convolves with the target image. When the operator is of a similar size to the target size, the response will be enhanced at the center area $(+)$ and suppressed at the surrounding area $(-)$. Therefore, by the operation of equation (6), the target center $\left(x_{c}, y_{c}\right)$ can be located at the maximum response after convolution, where * is the convolution operation:

$$
\begin{aligned}
\operatorname{LoG}(x, y, \sigma) & =\nabla^{2} G \\
& =-\frac{1}{\pi \sigma^{4}}\left[1-\frac{x^{2}+y^{2}}{2 \sigma^{2}}\right] e^{-\left(x^{2}+y^{2}\right) / 2 \sigma^{2}}, \\
G(x, y, \sigma) & =\frac{1}{2 \pi \sigma^{2}} e^{-\left(x^{2}+y^{2}\right) / 2 \sigma^{2}}, \\
\left(x_{c}, y_{c}\right) & =\max _{(x, y)} \nabla^{2} G * I_{p} .
\end{aligned}
$$

However, usually, the target size $\sigma$ is unknown. When there are multiple targets with different sizes that need to be detected, a single-scale LoG operator cannot meet the detection requirements. Multiscale representation is an effective method to address the problem [27]. Multiple scale 


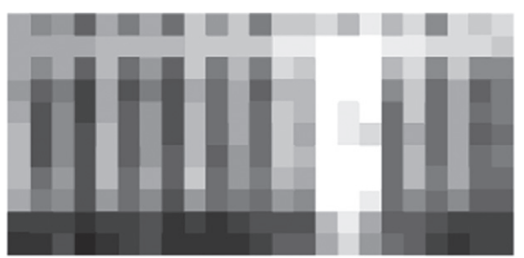

(a)

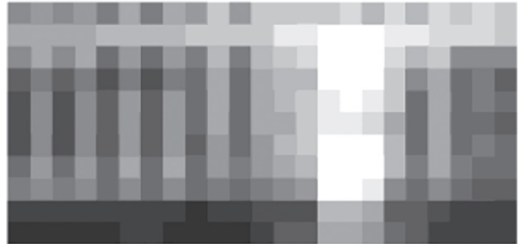

(b)

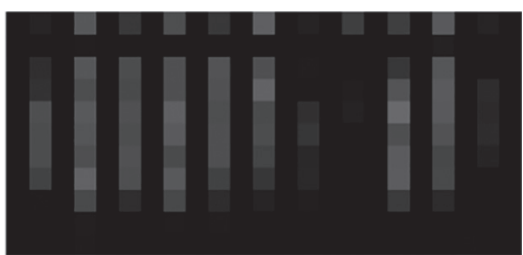

(c)

FIGURE 5: (a) Image patch of intercell busbar with heterogeneous background and a short circuit. (b) The estimated background. (c) Result of background removal. There is an opening among normal working suspenders.

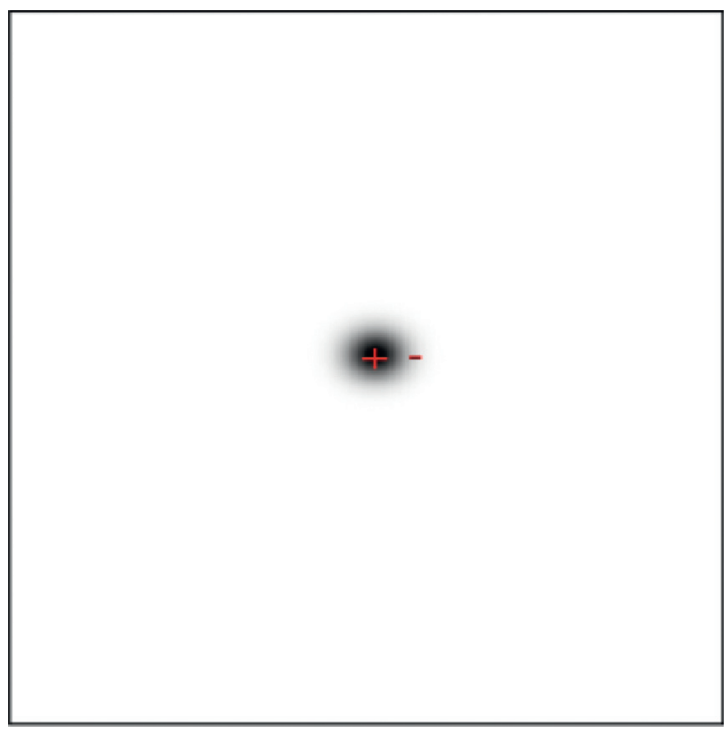

(a)

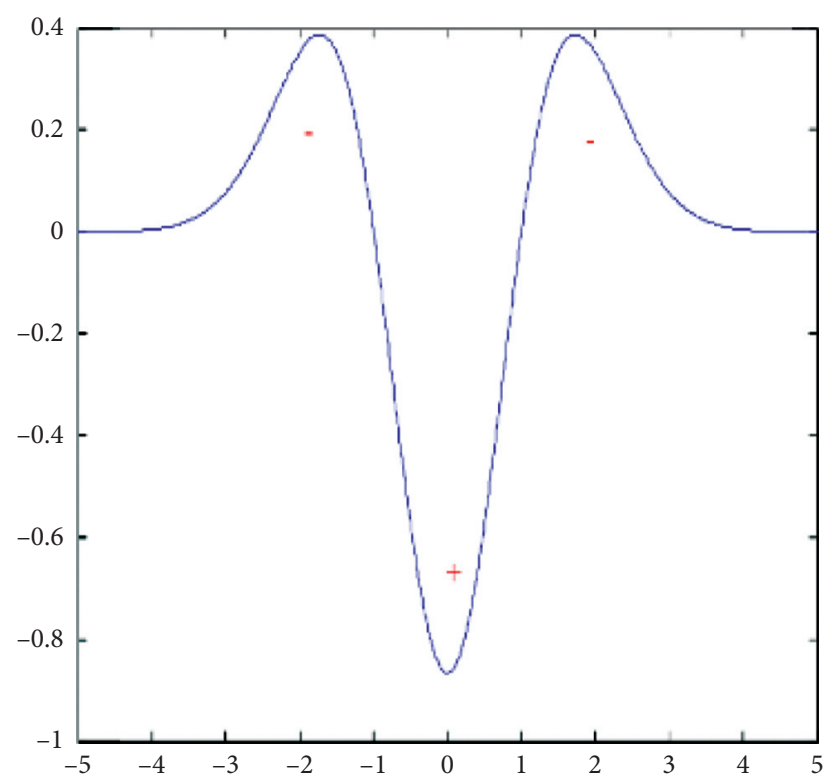

(b)

Figure 6: (a) Spot-like target. (b) One-dimensional Laplacian of Gaussian [23].

operators are used to construct a scale space. Searching for the largest response in a certain scale space can locate a target that is close to the scale of the space. Scale space $L(x, y, \sigma)$ is constructed by the convolution of a variable-scale Gaussian function with the input image $I_{p}(x, y)$ as the following equation:

$$
L(x, y, \sigma)=G(x, y, \sigma) * I_{p}(x, y) .
$$

In [28], the difference of two Gaussian functions (DoG) with different standard deviations is proved to be an approximation of the following scale-normalized LoG equations:

$$
\begin{aligned}
\operatorname{DoG}(x, y, \sigma) & =G(x, y, k \sigma)-G(x, y, \sigma), \\
\sigma \nabla^{2} G & =\frac{\partial G}{\partial \sigma} \\
& \approx \frac{G(x, y, k \sigma)-G(x, y, \sigma)}{k \sigma-\sigma} .
\end{aligned}
$$

Hence, the DoG scale space is constructed as equation (10). $k$ is a constant multiplicative factor, representing the scale change:

$$
\begin{aligned}
D(x, y, \sigma) & =(G(x, y, k \sigma)-G(x, y, \sigma)) * I_{p}(x, y) \\
& =L(x, y, k \sigma)-L(x, y, \sigma) .
\end{aligned}
$$

When the varied coordinates $(x, y)$ and $\sigma$ of DoG approach the central location $\left(x_{c}, y_{c}\right)$ and the radius of the target in the image, the target response is larger, and the local extremum is obtained. There is a candidate target at the position. Different size small targets detection problem is transformed from equation (6) to the following equation:

$$
\left(x_{c}, y_{c}, \sigma\right)=\max _{(x, y, \sigma)} \operatorname{DoG}(x, y, k \sigma) * I_{p} .
$$

After constructing the scale space, lots of candidate targets can be located by searching the maximum response. But there are many false targets as the DoG filter is sensitive to noise. SCR is an effective numerical criterion for screening candidate targets [23], and the calculating of the SCR is as equation (12). It is the quantitative calculation of the gray difference between the target and the local background:

$$
\operatorname{SCR}=\frac{\left|\mu_{T}-\mu_{B}\right|}{\sigma c},
$$


where $\mu_{T}$ and $\mu_{B}$ represent the gray intensity of the target and the local background, respectively. $\sigma_{c}$ denotes the standard deviation of the local background. Figure 7 shows targets with different SCR. The higher the SCR is, the better the contrast between the target and the local background is, and the more easily is the target to be recognized.

\subsubsection{Normal Working Suspender Detection with Improved} DoG Filter. The small target detection theory is based on a critical assumption that the target structure is isotropic Gaussian. It is acceptable in a particular condition. But in reality, the targets shapes are usually nonisotropic or deform on the image plane due to the optical effect. In our problem, normal suspenders are rectangular in shape and parallel to the coordinate axis of the image. Due to thermal diffusion and the inherent low resolution of infrared thermal imaging camera, the shape of the exposed suspender is elliptical, and equation (13) is used to model the structure:

$M\left(x, y \mid\left(x_{o}, y_{o}, \sigma_{x}, \sigma_{y}\right)\right)=A \exp \left\{-\left(\frac{\left(x-x_{o}\right)^{2}}{2 \sigma_{x}^{2}}+\frac{\left(y-y_{o}\right)^{2}}{2 \sigma_{y}^{2}}\right)\right\}$,

$\left(x_{o}, y_{o}\right)$ is the target central position. $A$ is the peak intensity which can automatically be determined after detecting the central position. Here, we set two scale parameters $\sigma_{x}, \sigma_{y}$ and consider that these two scale parameters are not related. It is because of the rigid nature of the hanging ear. The length may vary with the cover operation, but the width is fixed. Correspondingly, the nonisotropic Gaussian functions equation (14) is used to construct matching filters:

$$
G\left(x, y, \sigma_{x}, \sigma_{y}\right)=\frac{1}{2 \pi \sigma_{x} \sigma_{y}} e^{-\left(\left(x^{2} / 2 \sigma_{x}^{2}\right)+\left(y^{2} / 2 \sigma_{y}^{2}\right)\right)} .
$$

Like equation (9), the corresponding LoG filter which is the second derivative of the Gaussian is approximately the derivative about the scale:

$$
\left[\sigma_{x}, \sigma_{y}\right]^{T}\left[\frac{\partial^{2} G}{\partial \sigma_{x}^{2}}, \frac{\partial^{2} G}{\partial \sigma_{y}^{2}}\right] \approx\left[\frac{\partial G}{\partial \sigma_{x}}, \frac{\partial G}{\partial \sigma_{y}}\right],
$$

where $\sigma_{x}$ is a constant. Utilizing the separability of convolution, we only change scale $\sigma_{y}$ in constructing DoG scale space:

$$
\begin{aligned}
D\left(x, y, \sigma_{x}, \sigma_{y}\right) & =\left(G\left(x, y, \sigma_{x}, k \sigma_{y}\right)-G\left(x, y, \sigma_{x}, \sigma_{y}\right)\right) * I_{p}(x, y) \\
& =L\left(x, y, \sigma_{x}, k \sigma_{y}\right)-L\left(x, y, \sigma_{x}, \sigma_{y}\right),
\end{aligned}
$$$$
\left(x_{o}, y_{o}, \sigma_{y}\right)=\max _{\left(x, y, \sigma_{y}\right)} D\left(x, y, \sigma_{x}, \sigma_{y}\right) .
$$

Since the scale of width $\sigma_{x}$ is a constant, change the length scale $\sigma_{y}$ to build scale space, and the space from the original three dimensions reduced to two, which greatly reduces the difficulty of extreme value search. Figure 8 shows the detection result of Figure 5(c) with the improved DoG filter. There is an opening with a large lateral distance between the two detected normal suspenders. We considered it a suspicious short circuit area.

3.2.3. Suspicious Short Circuit Area Confirmed. A numerical standard about the lateral distance is still required to recognize the suspicious short circuit area. Texture feature is an important feature in industrial vision [29]. Considering the busbar area presents regular texture, the period of the textures can be used as the criterion. The regular texture is characterized by repeated patterns of a structure unit, which means that the pixel intensities in the structure are highly spatial related. The gray level cooccurrence matrix (GLCM) [30] was proposed to describe the spatial correlation of gray intensities, and the correlation descriptor of the GLCM is the quantitative calculation of the linear dependence of two pixels.

In the busbar area, pixels intensities are repeating in equal intervals along the horizontal. A transverse structure is defined as $Q$ (Figure 9). The structure describes a relative position of two pixels with " $t$ pixel offset on the right." The correlation of the two pixels in the structure with an offset $t$ is calculated according to

$$
\operatorname{cor}_{t}=\sum_{i=1}^{k} \sum_{j=1}^{k} \frac{\left(i-m_{\mathrm{tr}}\right)\left(j-m_{\mathrm{tc}}\right) G_{t}(i, j)}{\sigma_{\mathrm{tr}} \sigma_{\mathrm{tc}}},
$$

where $G_{t}(k \times k)$ is the computed gray cooccurrence matrix according to $Q$ and $k=255$ is the dimension of $G$. $m_{\mathrm{tr}}$ and $m_{\mathrm{tc}}$ are the means of $G_{\mathrm{tr}}(i)=\sum_{j=1}^{k} G_{t}(i, j)$ and $G_{\mathrm{tc}}(j)=\sum_{i=1}^{k} G_{t}(i, j)$, respectively, and $\sigma_{\mathrm{tr}}$ and $\sigma_{\mathrm{tc}}$ are the corresponding standard deviation.

Changing the offset value $t$, we can compute a sequence of gray cooccurrence matrix $G_{t}(k \times k)$, where $t$ is an integer and the value range is $[1,2, \ldots, 16]$. The correlation curve of structures for different transverse offsets is as shown in Figure 10. The curve presents a repetitive model; the correlation value is larger at an even offset. Though the correlation values at the even offsets are not absolutely equal, the repetitive pattern reveals that the texture period is 2 pixels.

Compute the transverse distance between two adjacent detected normal suspenders and compare it with the estimated period of texture. When the computed transverse distance is larger than the period, the corresponding area is a suspicious short circuit area. The number of suspenders contained in a suspicious area is calculated by equation (19). $n$ is the suspender number. $d_{\text {trans }}$ is the pixel distance of the suspicious short circuit area, and $T_{\text {trans }}$ is the transverse period:

$$
n=\left\lceil\frac{d_{\text {trans }}}{T_{\text {trans }}}\right\rceil .
$$

\section{Experiment and Results}

4.1. Fault Sensitivity Comparison between Busbar Area and Cloth Area. To explain that the intercell busbar area is more suitable for short circuit detection than the cloth area, we 


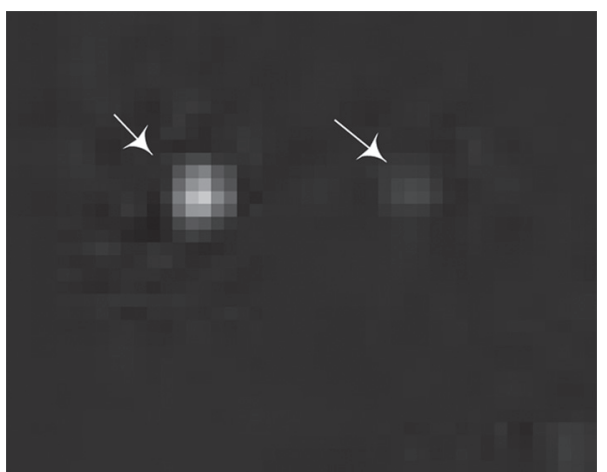

(a)

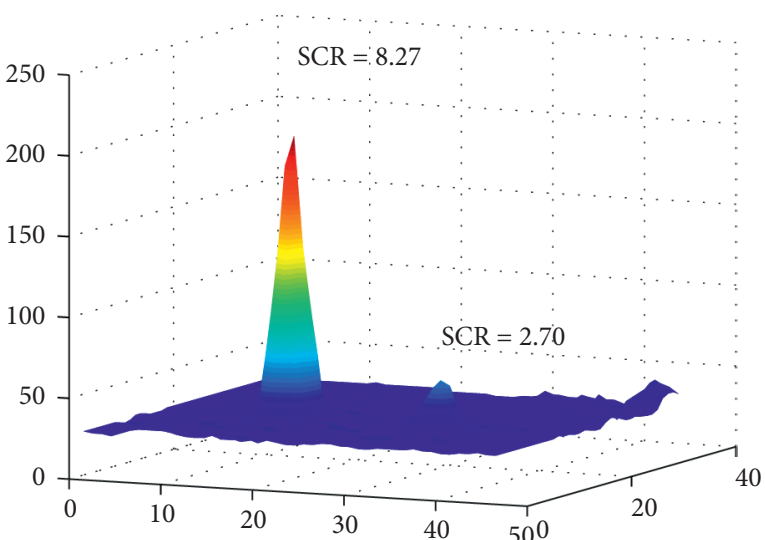

(b)

FIgURE 7: (a) Image with two small targets. (b) SCR of the two small targets in 3D view.

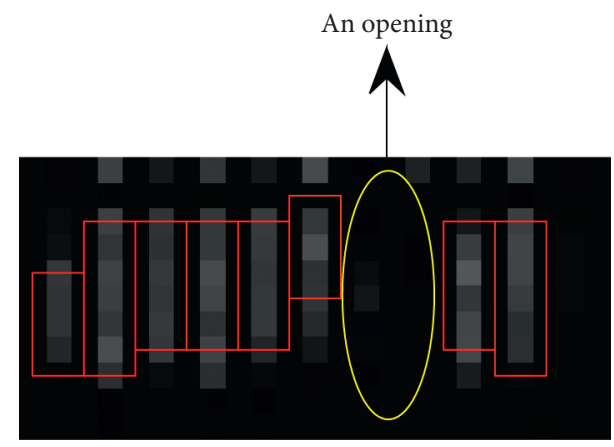

Figure 8: The detection result of Figure 5(c) by the improved DoG filter.

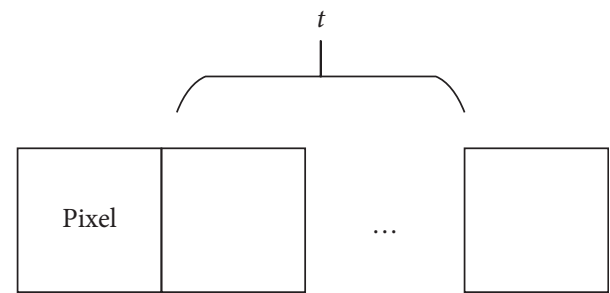

FIgURE 9: Transverse structure (Q) depicts the repetitive structure of the transverse regular texture.

utilize a commonly used diagnostic method, the gray-scale longitudinal accumulation of the two areas, to diagnose a short circuit. Figure 11 shows an example IR image; the marked electrolytic cell contains four short circuits which have been diagnosed manually.

Figure 12 represents the gray accumulation curves of the two areas. From the curve of the cloth area, three peaks can be diagnosed as short circuits by a threshold. But short circuit 4 is missed because the corresponding temperature did not rise significantly. Many factors can lead to this phenomenon such as the cover cloth is not in contact with the electrode, spray water, and short failure time. From the curve of the intercell busbar, four peaks can be diagnosed as

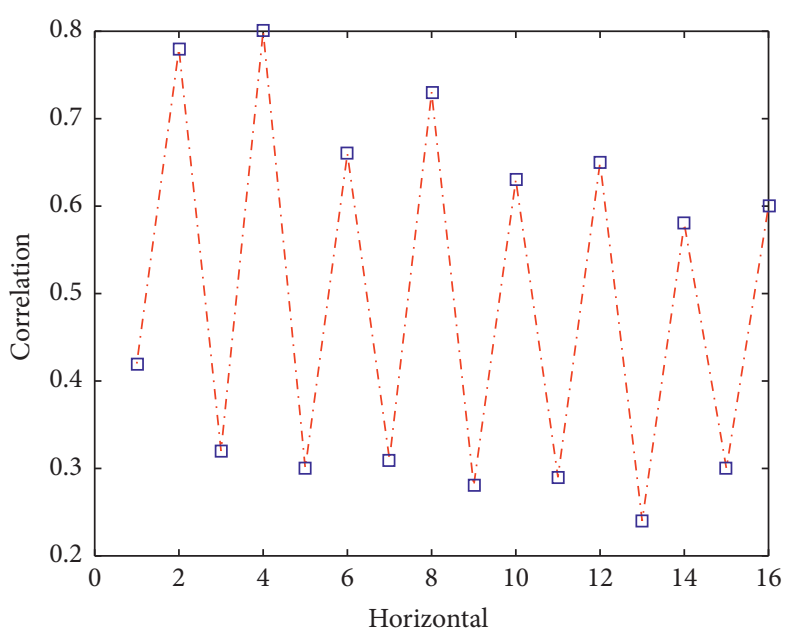

FIGURE 10: Correlation values of the structure as a function of the transverse offset.

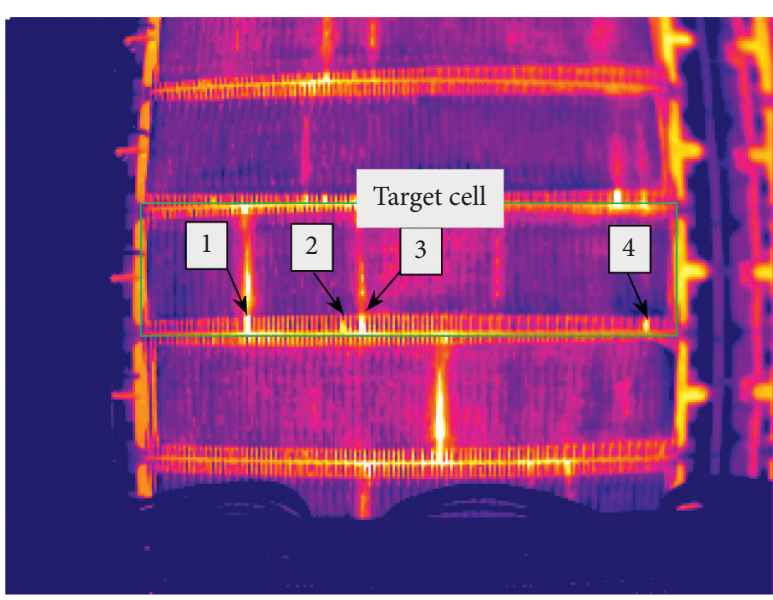

FIgURE 11: Target cell in an IR image.

short circuits, though the diagnostic information provided by the intercell busbar is disturbed by more severe clutter. 


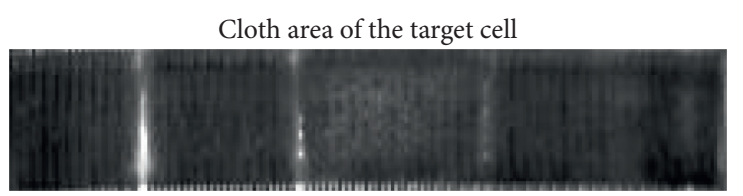

(a)

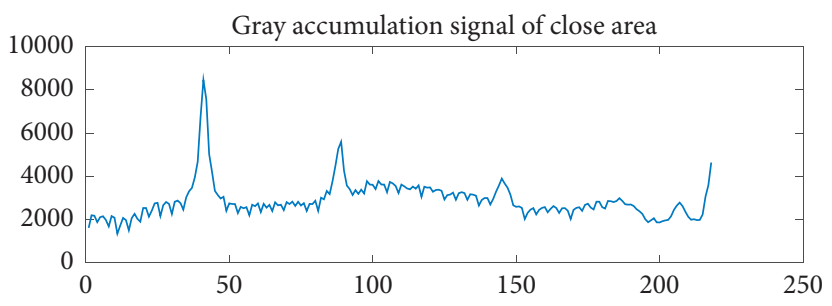

(c)

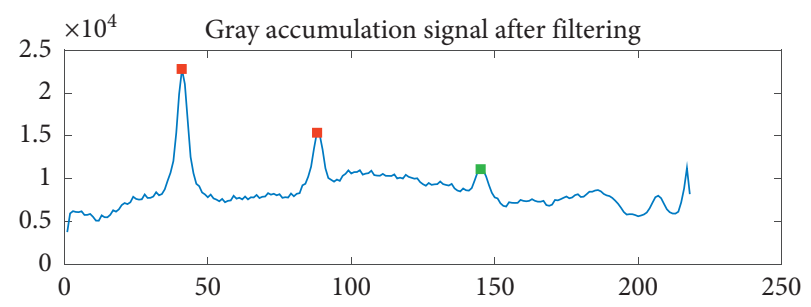

(e)
Intercell busbar area of the target cell

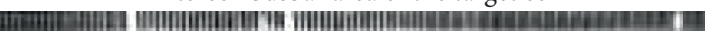

(b)

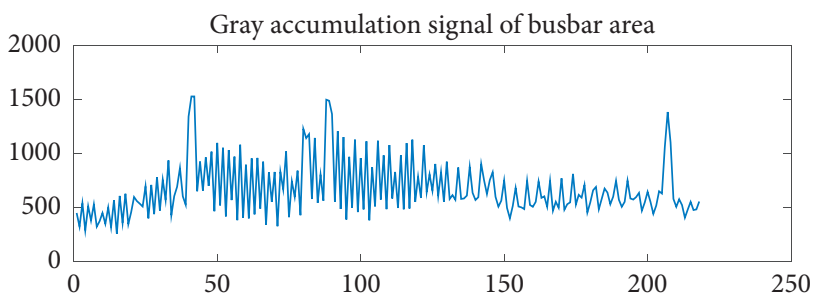

(d)

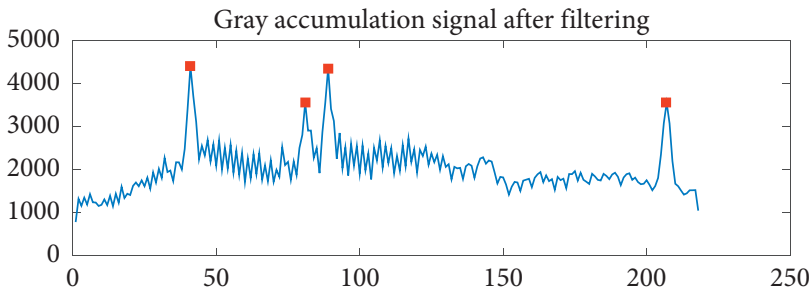

(f)

Figure 12: Gray accumulation signal of the cloth area and the intercell busbar area.

\subsection{Comparison of Normal Suspender Detection Accuracy of} Different Methods. Normal suspender detection is the key step of the proposed two-level short circuit detection scheme. We compare the detection performance of the proposed orthogonal DoG filter with the DoG filter [23] and the top-hat filter since the two methods have achieved good performance in detecting small targets. The experiments are executed on the intercell busbar of the target cell in Figure 11. There are 230 suspenders on the busbar, and the sequence numbers of the four short circuits have been manually identified in advance. Precision and recall rate of the normal suspender are adopted as the evaluation metrics:

$$
\begin{aligned}
& \text { precision }=\frac{\mathrm{TPn}}{\mathrm{NDt}}, \\
& \text { recallrate }=\frac{\mathrm{TPn}}{\mathrm{ACn}},
\end{aligned}
$$

where TPn is the number of true positive normal suspenders; NDt is the number of detected targets; and ACn is the actual number of normal suspenders in the cell.

After the same background removal preprocessing, we use the three methods to detect the normal working suspenders. When we use the orthogonal DoG filter to construct scale space, the horizontal scale parameter is a constant $\sigma_{x}=0.4$, while $\sigma_{y}$ varies as defined in equation (16). The same as the value set in [28], $k=1.2$ and the number of scale space image is 8 . In the DoG filter method, we set $\sigma_{x}=\sigma_{y}=\sigma=0.5$, and the scale parameter changes according to equation (10).
Figure 13 shows the enlarged view of the initial detection results. For convenience, we mark an asterisk on each detection target. More normal suspenders are detected by the two DoG methods than those of the top-hat method, especially on the right blurry area. That is because the top-hat method involves morphological operation, so a binary image is needed. To obtain the binary image, a gray threshold is needed. After the background is removed, the gray intensity of the target in the blurred area is too weak to be ignored by the threshold operation. The DoG detection methods can enhance the contrast while matching the target gray intensity distribution pattern. Therefore, in the blurry area, the DoG methods show better detection ability than the top-hat method. The orthogonal DoG filter detects more targets than the original DoG filter, which can better meet the needs of short circuit detection. The same suspender is repeatedly detected, and this phenomenon can be found in both methods. But with the orthogonal DoG filter, this situation will be more serious. Because the varying longitudinal scale $\sigma_{y}$ is sensitive to the gray scale continuity of a suspender. When estimating the background, a horizontal median filter is used, which inevitably changes the gray value of the suspender, resulting in longitudinal discontinuity of gray intensity after subtracting the background. The same suspender is considered by the filter as two detection targets. It will reduce the precision of the orthogonal DoG method.

An SCR threshold 4 is applied to filter candidate targets and eliminate noise targets. The final detection results are shown in Figure 14. The orthogonal DoG filter detects 241 targets. The number of that with the DoG filter is 131. Table 1 summarizes the statistical results in terms of the recall rate 

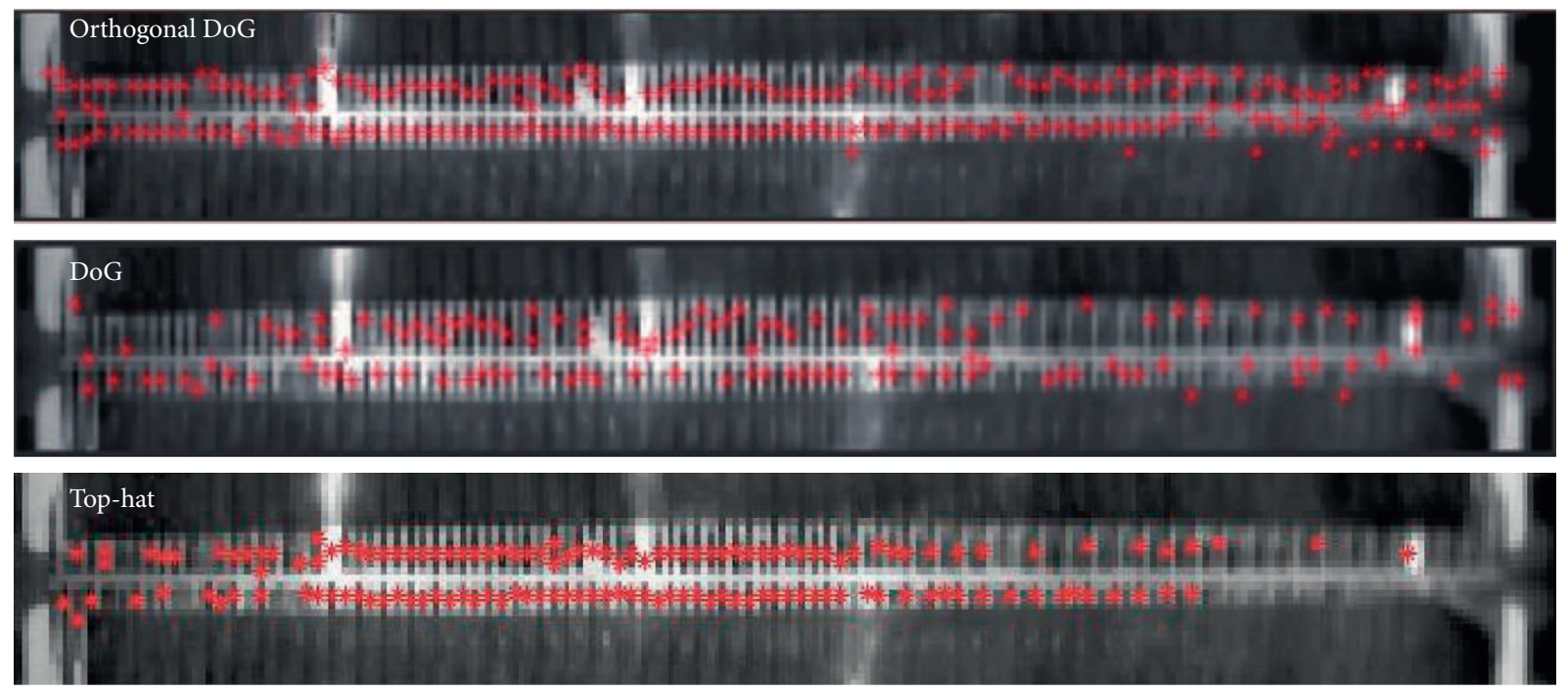

FIGURE 13: Candidate targets with the improved orthogonal DoG filter, DoG filter, and the top-hat filter. (a) Orthogonal DoG. (b) DoG. (c) Top-hat.
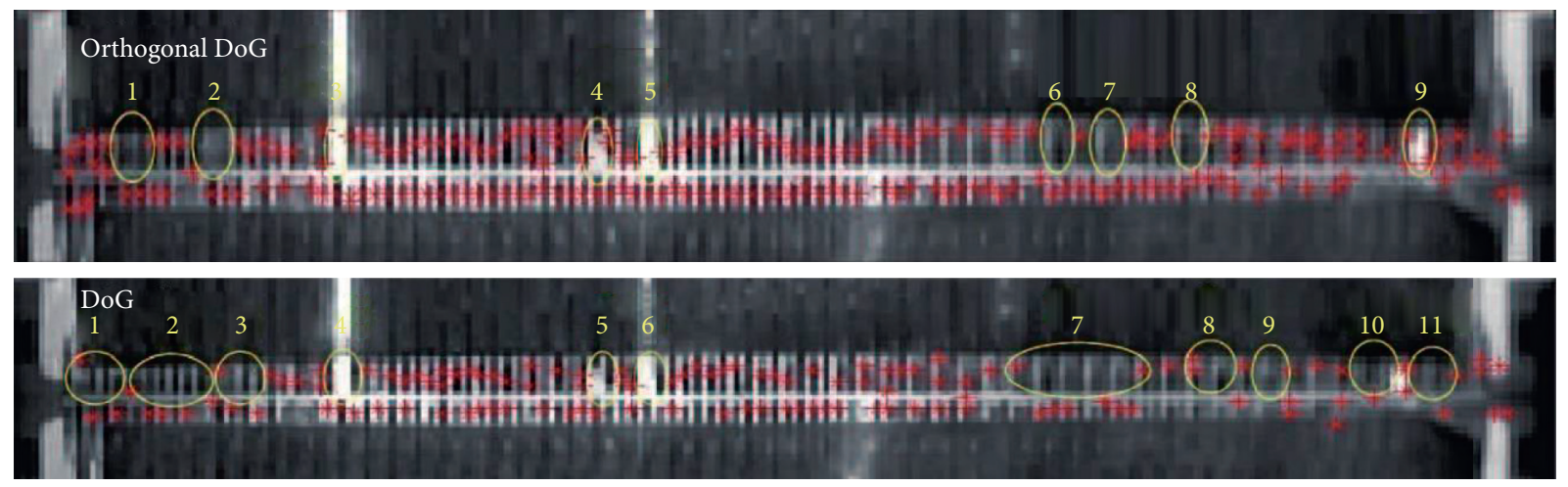

FIGURE 14: Final detection result and suspected short circuit areas with the improved orthogonal DoG filter and the DoG filter. (a) Orthogonal DoG. (b) DoG.

TABLE 1: Statistical comparison of normal suspender detection between the improved orthogonal DoG filter and DoG filter.

\begin{tabular}{lcc}
\hline Criteria & DoG & Orthogonal DoG \\
\hline Recall rate & $46.9 \%$ & $85.8 \%$ \\
Precision & $80.9 \%$ & $80.5 \%$ \\
Total detection numbers & 131 & 241 \\
\hline
\end{tabular}

and precision. Though the precision with the DoG filter is 0.4 higher than that with the orthogonal DoG filter, it is within an acceptable range. The orthogonal DoG outperforms the DoG filter with respect to the recall rate, which means that the proportion of the accurately detected normal suspenders is high. This is due to the fact that changing scale parameters $\sigma_{y}$ can better match the intensity distribution pattern of the suspender.

4.3. Results and Discussion of the Accuracy for Short Circuit Detection. The ultimate purpose of normal suspender detection is to locate suspicious short circuit areas and further identify the real short circuits. Base on the period of the texture, the circle areas in Figure 14 are selected as suspicious short circuit areas that belong to the target cell. There are nine suspicious short circuit areas obtained with the improved orthogonal DoG method, while the number of that with the DoG filter is eleven. Although the four known short circuits are all included in the suspicious short circuit areas determined by the two methods, it is obvious that the lateral distances of the suspicious short circuit areas obtained by the orthogonal DoG method are smaller. It demonstrates that the improved orthogonal DoG method can provide less uncertainty for real short circuit identification. Region 1, region 2 , region 6 , region 7 , and region 8 identified by the orthogonal DoG method are areas where the blur is serious. The blur is caused by uneven current distribution among electrodes. The currents on the electrode of these areas are larger than normal and may further trigger a short circuit; thus, these areas need to be inspected frequently. According to the period of the texture and the horizontal distance of the suspicious short circuit areas (equation (19)), the number of electrodes in each suspicious short circuit area is calculated. 
TABLE 2: The number of suspenders contained in each suspicious short circuit area.

\begin{tabular}{lcc}
\hline Suspicious area & Suspender number (orthogonal DoG) & Suspender number (DoG) \\
\hline 1 & 3 & 3 \\
2 & 3 & 6 \\
3 & 1 & 3 \\
4 & 1 & 2 \\
5 & 1 & 2 \\
6 & 2 & 2 \\
7 & 3 & 7 \\
8 & 2 & 3 \\
9 & 1 & 3 \\
10 & & 3 \\
\hline
\end{tabular}

TAвLe 3: Serial numbers of the four short circuits determined by the orthogonal DoG filter and the DoG filter.

\begin{tabular}{|c|c|c|c|c|}
\hline Real short circuit & 1 & 2 & 3 & 4 \\
\hline Serial number (orthogonal DoG) & $\# 10$ & $\# 21$ & $\# 23$ & $\# 47$ \\
\hline Serial number (DoG) & $\# 10$ & $\# 18$ & $\# 20$ & $\# 40$ \\
\hline Real serial number & $\# 9$ & $\# 20$ & \#22 & \#51 \\
\hline
\end{tabular}

TABLE 4: Basic technical parameters of the thermal camera.

\begin{tabular}{lc}
\hline Items & Technical parameters \\
\hline Detector type & Uncooled focal plane array (UFPA) \\
Infrared band & $8 \sim 14 \mathrm{~m}$ \\
Pixel & $240 \times 320$ \\
Field angle & $25^{\circ} \times 19^{\circ} \mathrm{C}$ \\
Measuring range & $-25^{\circ} \mathrm{C} \times 650^{\circ} \mathrm{C}$ \\
Thermal sensitivity & $\pm 2^{\circ} \mathrm{C}$ \\
Frame rate & $50 / 60 \mathrm{~Hz}$ \\
\hline
\end{tabular}

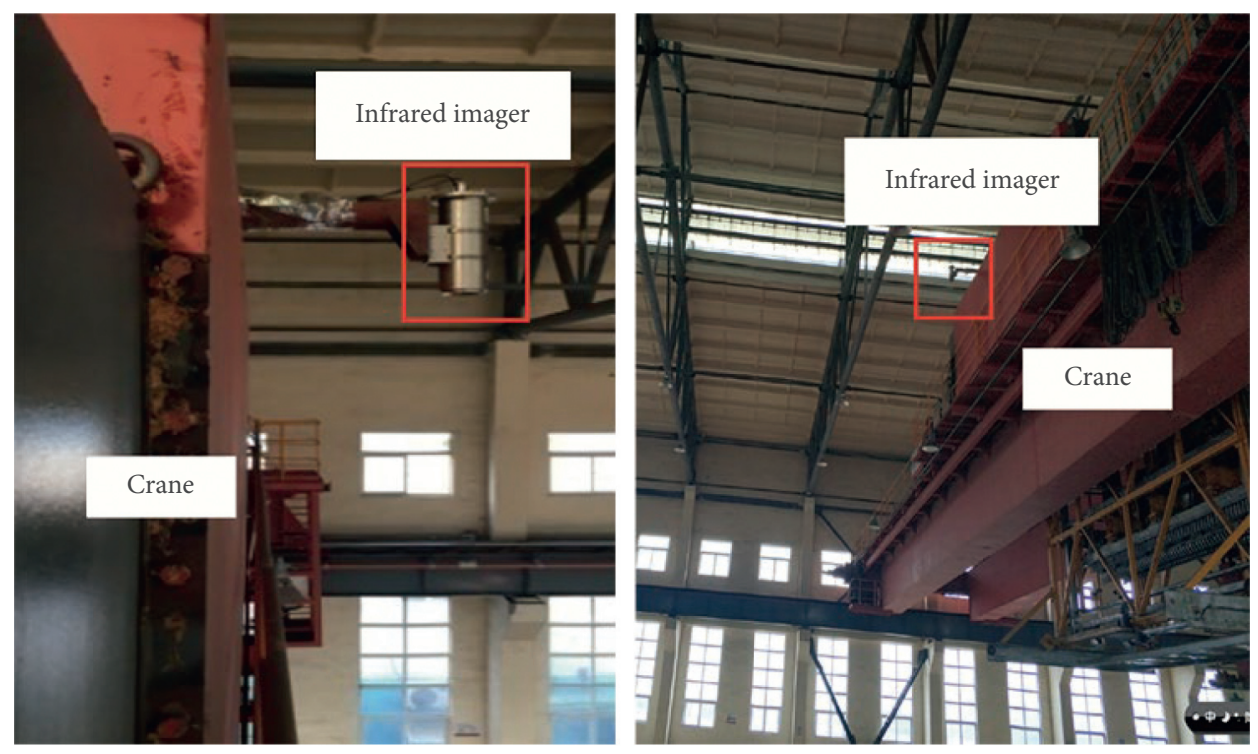

Figure 15: Infrared camera mounted on the crane.

The number of electrodes contained in each suspicious short circuit area is shown in Table 2.

The determined serial numbers of the four real short circuits with the two methods are shown in Table 3. With the improved orthogonal DoG filter, the detected short circuits serial numbers are closer to real serial numbers. But due to the accumulation of errors, the precision will decrease. So, the deviation of the serial number of short circuit 4 is larger 
TABLe 5: Statistical result of the detection system.

\begin{tabular}{lc}
\hline Category & Statistical number \\
\hline Cell number & 234 \\
Cathode number & 13338 \\
Real short circuit & 75 \\
Detected short circuit & 175 \\
Short circuit with high temperature & 76 \\
Error detection of high temperature short circuit & 13 \\
Potential short circuit & 99 \\
Error detection of potential short circuit & 87 \\
\hline
\end{tabular}

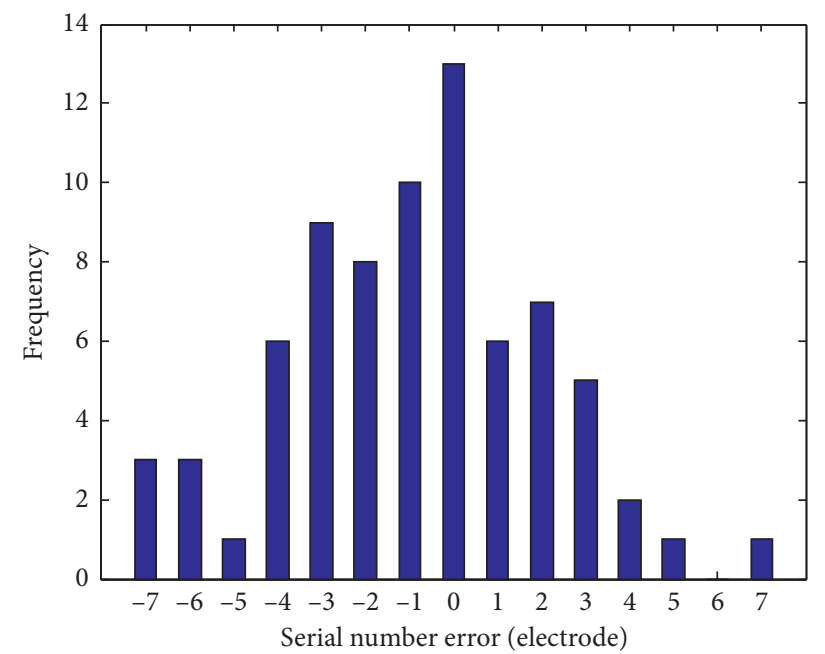

Figure 16: Histogram of serial number error of grand-truth short circuits.

than others. The orthogonal DoG filter is superior to the DoG filter in providing higher precision for short circuit serial number confirmation.

4.4. Results and Discussion of On-Site Application. The detection system was verified at a copper electrolysis plant in China. Details of the thermal camera are as shown in Table 4. The camera is mounted vertically down on top of the crane and can be moved with the crane, with a distance of $8.3 \mathrm{~m}$ from the lens to the cell surface (Figure 15).

The detection system continuously detected 234 cells whose surface is covered with cloth. There are 57 cathode electrodes in each cell, and a total of 75 short circuits have been identified manually in advance. The detection statistical results are shown in Table 5.

The system detected 76 apparent high temperature short circuits, 13 of which were fault-detected, and these misdetected electrodes were short circuited electrodes that had been excluded, although their temperature had not returned to normal. 99 potential short circuits were diagnosed, of which 87 were detected by error. The main reason for the high error detection is that the uneven current distribution leads to an increase in the number of blurry areas on the busbar, which are diagnosed as suspicious short circuit areas. Although this operation reduces the accuracy of the detection, it effectively avoids missed detection and reduce the influence time of a low electrolytic efficiency state.
These real short circuits are randomly distributed in electrolytic cells. The histogram of serial number error distributions of these short circuit is shown in Figure 16. The maximum absolute error was 7 electrodes, but short circuits with an error of fewer than 4 electrodes account for $77.3 \%$. This means that the serial number of the vast majority of short circuited electrodes can be precisely located, which reduces the workload of staff in subsequent short circuit removal.

\section{Conclusion}

In this research, we design a two-level short circuit detection scheme for copper electrorefining based on the infrared image. After analyzing the cells circuit and thermal distribution characteristics, the intercell busbar region is used to perform short circuit detection. In order to solve the problem that short circuited electrodes are against highthermal background, in the first step, short circuited electrodes are regarded as parts of the background due to the close gray intensities. Background subtraction is introduced. By removing background as well as short circuited electrodes, normal working electrodes can be extracted from the high intensity background. At the same time, an opening is formed at the position of the short circuited electrode. In the second step, normal working electrodes are detected with an improved DoG filter firstly. Then, considering the texture period of the busbar area, a suspicious short circuit area can 
be identified by comparing the distance between two detected targets and the estimated texture period. In this step, we improve the scale parameter of the DOG filter according to the electrode shape characteristics and use the separability of Gaussian convolution to calculate, which greatly improves the detection rate of normal working electrodes and provides a guarantee for suspicious short circuit area detection. Benefiting from this scheme, we can effectively avoid short circuit missed detection and help workers to concentrate on cells maintenance.

\section{Data Availability}

The data used to support the findings of this study are available from the corresponding author upon request.

\section{Conflicts of Interest}

The authors declare that they have no conflicts of interest.

\section{Acknowledgments}

This work was supported by the National Natural Science Foundation of China (Grant nos. 61673400 and 61890930-2) and the Postgraduate Research Innovation Project of Central South University (Grant no. 502221809).

\section{References}

[1] P. E. Aqueveque, E. P. Wiechmann, and R. P. Burgos, "Shortcircuit detection for electrolytic processes employing optibar intercell bars," IEEE Transactions on Industry Applications, vol. 45, no. 4, pp. 1225-1231, 2009.

[2] E. L. Loboda, I. S. Anufriev, M. V. Agafontsev et al., "Evaluating characteristics of turbulent flames by using IR thermography and PIV," Infrared Physics \& Technology, vol. 92, pp. 240-243, 2018.

[3] S. B. Borg, "Infrared detection of invisible shorts in electrolytic copper refining tanks," IEEE Transactions on Industrial Electronics and Control Instrumentation, vol. IECI-18, no. 2, pp. 36-37, 1971.

[4] M. Esa, T. T. Juha, and V. Henri, "IR-based system for shortcircuit detection during copper electrorefining process," Proceedings of SPIE-The International Society for Optical Engineering, vol. 3652, pp. 2-9, 1999.

[5] Y. Li, Y. Zhang, A. Geng, L. Cao, and J. Chen, "Infrared image enhancement based on atmospheric scattering model and histogram equalization," Optics \& Laser Technology, vol. 83, pp. 99-107, 2016.

[6] G. Morris and P. Angelov, "Real-time novelty detection in video using background subtraction techniques: state of the art a practical review," IEEE International Conference on Systems, Man and Cybernetics, pp. 537-543, 2014.

[7] S. Kim, Y. Yang, J. Lee, and Y. Park, "Small target detection utilizing robust methods of the human visual system for IRST," Journal of Infrared, Millimeter, and Terahertz Waves, vol. 30, no. 9, pp. 994-1011, 2009.

[8] X. Bai and F. Zhou, "Analysis of new top-hat transformation and the application for infrared dim small target detection," Pattern Recognition, vol. 43, no. 6, pp. 2145-2156, 2010.

[9] P. Wang, J. W. Tian, and C. Q. Gao, "Infrared small target detection using directional highpass filters based on LSSVM," Electronics Letters, vol. 45, no. 3, pp. 156-158, 2009.
[10] E. P. Wiechmann, G. A. Vidal, and A. J. Pagliero, "Currentsource connection of electrolytic cell electrodes: an improvement for electrowinning and electrorefinery," IEEE Transactions on Industry Applications, vol. 42, no. 3, pp. 851-855, 2006.

[11] E. P. Wiechmann, L. G. Munoz, P. E. Aqueveque, G. A. Vidal, and J. A. Henriquez, "Introducing a bypass-backup connection system for current-mode copper electrowinning intercell bars," IEEE Transactions on Industry Applications, vol. 50, no. 2, pp. 1490-1495, 2014.

[12] E. P. Wiechmann, A. S. Morales, P. E. Aqueveque, J. A. Henriquez, and L. G. Munoz, "3D FEM thermal and electrical analysis of copper electrowining intercell bars," in Proceedings of the 2015 IEEE Industry Applications Society Annual Meeting, pp. 1-7, Addison, TX, USA, October 2015.

[13] R. Zhao, L. Lin, H. Li, Y. Wang, and J. Tie, "A new approach to establishing cathode rod temperature-current model based on nickel electrolysis cells," Sensors \& Transducers Journal, vol. 168, pp. 249-259, 2014.

[14] E. P. Wiechmann, P. Aqueveque, G. A. Vidal, and J. A. Henriquez, "Contact system design to improve energy efficiency in copper electrowinning processes," IEEE Transactions on Industry Applications, vol. 49, no. 6, pp. 2461-2465, 2013.

[15] W. Wang, C. Li, and J. Shi, "A robust infrared dim target detection method based on template filtering and saliency extraction," Infrared Physics \& Technology, vol. 73, pp. 19-28, 2015.

[16] K. Zhang and X. Li, "Infrared small dim target detection based on region proposal," Optik, vol. 182, pp. 961-973, 2019.

[17] S. D. Deshpande, H. E. Meng, R. Venkateswarlu, and P. Chan, "Max-mean and max-median filters for detection of small targets," Signal and Data Processing of Small Targets, vol. 3809, 1999.

[18] Y. Gu, C. Wang, B. Liu, and Y. Zhang, "A kernel-based nonparametric regression method for clutter removal in infrared small-target detection applications," IEEE Geoscience and Remote Sensing Letters, vol. 7, no. 3, pp. 469-473, 2010.

[19] C. L. P. Chen, H. Li, Y. Wei, T. Xia, and Y. Y. Tang, "A local contrast method for small infrared target detection," IEEE Transactions on Geoscience \& Remote Sensing, vol. 52, no. 1, pp. 574-581, 2013.

[20] Y. Li and Y. Zhang, "Robust infrared small target detection using local steering kernel reconstruction," Pattern Recognition, vol. 77, pp. 113-125, 2018.

[21] X. Wang, Z. Peng, D. Kong, and Y. He, "Infrared dim and small target detection based on stable multisubspace learning in heterogeneous scene," IEEE Transactions on Geoscience and Remote Sensing, vol. 55, no. 10, pp. 5481-5493, 2017.

[22] M. Nasiri, M. R. Mosavi, and S. Mirzakuchaki, "IR small target detection based on human visual attention using pulsed discrete cosine transform," IET Image Processing, vol. 11, no. 6, pp. 397-405, 2017.

[23] S. Kim and J. Lee, "Scale invariant small target detection by optimizing signal-to-clutter ratio in heterogeneous background for infrared search and track," Pattern Recognition, vol. 45, no. 1, pp. 393-406, 2012.

[24] S. Moradi, P. Moallem, and M. F. Sabahi, "Scale-space point spread function based framework to boost infrared target detection algorithms," Infrared Physics \& Technology, vol. 77, pp. 27-34, 2016.

[25] Z. Miao and X. Jiang, "Interest point detection using rank order LoG filter," Pattern Recognition, vol. 46, no. 11, pp. 2890-2901, 2013. 
[26] X. Dong, X. Huang, Y. Zheng, L. Shen, and S. Bai, "Infrared dim and small target detecting and tracking method inspired by Human Visual System," Infrared Physics \& Technology, vol. 62 , pp. 100-109, 2014.

[27] T. Lindeberg, "Feature detection with automatic scale selection," International Journal of Computer Vision, vol. 30, no. 2, pp. 79-116, 1998.

[28] D. G. Lowe, "Distinctive image features from scale-invariant keypoints," International Journal of Computer Vision, vol. 60, no. 2, pp. 91-110, 2004

[29] Z. Chen, M. Lu, Y. Zhou, and C. Chen, "Information synergy entropy based multi-feature information fusion for the operating condition identification in aluminium electrolysis," Information Sciences, vol. 548, pp. 275-294, 2021.

[30] R. M. Haralick, K. Shanmugam, and I. H. Dinstein, "Textural features for image classification," IEEE Transactions on Systems, Man, and Cybernetics, vol. SMC-3, no. 6, pp. 610-621, 1973. 